\title{
Flavor Dependence of T-odd PDFs
}

\author{
Leonard P. Gamberg ${ }^{1}$, Gary R. Goldstein ${ }^{2}$, and Marc Schlegel ${ }^{3}$ \\ 1- Penn State University-Berks -Department of Physics and Astronomy \\ Reading, PA 19610 - USA \\ 2- Tufts University - Department of Physics and Astronomy \\ Medford, MA 02155 - USA \\ 3- Thomas Jefferson National Accelerator Facility - Theory Division \\ Newport News, VA 23608- USA
}

\begin{abstract}
The flavor dependence of the naive time reversal odd ("T-odd") parton distributions for $u$ - and $d$-quarks are explored in the spectator model. The flavor dependence of $h_{1}^{\perp}$ is of significance for the analysis of the azimuthal $\cos (2 \phi)$ asymmetries in unpolarized SIDIS and DY-processes, as well as for the overall physical understanding of the distribution of transversely polarized quarks in unpolarized nucleons. As a by-product of the formalism, we calculate the chiral-odd but "T-even" function $h_{1 L}^{\perp}$ which enables us to present a prediction for the single spin asymmetry $A_{U L}^{\sin (2 \phi)}$ for a longitudinally polarized target in SIDIS.
\end{abstract}

Naive time reversal-odd ("T-odd") transverse momentum dependent (TMD) parton distributions (PDFs) have gained considerable attention in recent years. Theoretically they can account for non-trivial transverse spin and momentum correlations such as single spin asymmetries (SSA) in hard scattering processes when transverse momentum scales are on the order of that of quarks in hadrons, namely $P_{T} \sim k_{\perp} \ll \sqrt{Q^{2}}$. A prominent example is the Sivers function $f_{1 T}^{\perp}[2]$ which explains the observed SSA in semi-inclusive deep inelastic scattering (SIDIS) for a transversely polarized proton target by the HERMES collaboration [3]. It describes correlations of the intrinsic quark transverse momentum and the transverse nucleon spin. The corresponding SSA on a deuteron target measured by COMPASS [4] vanishes, indicating a flavor dependence of the Sivers function. Another leading twist "T-odd" parton distribution, the chiral-odd Boer-Mulders function $h_{1}^{\perp}[5]$ correlates the transverse spin of a quark with its transverse momentum within the nucleon. We focus on the flavor dependence of these "T-odd" functions where for example $h_{1}^{\perp}$ is important for the analysis of the azimuthal $\cos (2 \phi)$ asymmetry in unpolarized SIDIS and Drell-Yan [6, 7]. We also consider the flavor dependence of the "T-even" function $h_{1 L}^{\perp}$, which is of interest in the transverse momentum and quark spin correlations in a longitudinally polarized target [8].

Considerable understanding of TMDs and fragmentation functions $(\mathrm{FF})$ have been gained from model calculations using the spectator framework $[9,10,6,7,11,12]$.

In this formalism we start (cf. [13]) from the definition of the unintegrated color gauge invariant quark-quark correlator which contains the gauge link indicated by the Wilson line, $\mathcal{W}[a \mid b]$, and work in Feynman gauge in which the transverse Wilson line vanishes [14]. In the diquark model the sum over the complete set of intermediate on-shell states in the definition of the correlator is represented by a single one-particle diquark state $\left|d q ; p_{d q}, \lambda\right\rangle$, where $p_{d q}$ is the diquark momentum and $\lambda$ its polarization. The diquark is "built" from two valence quarks which can be scalar-spin 0 or axial vector-spin 1 . The unintegrated correlator is then 


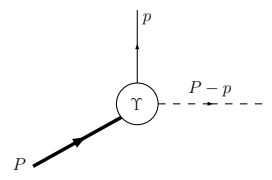

(a) Nucleon-

Diquark-

Quark vertex

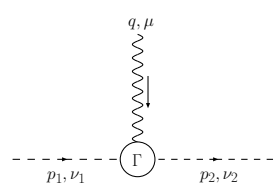

(b) Diquarkgluon vertex

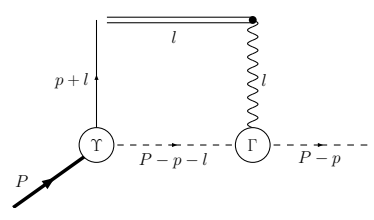

(c) Box-graph hermitian conjugated

Figure 1: Different vertices for the axial-vector diquark and contribution of the gauge link in the one-gluon approximation.

given by

$$
\begin{aligned}
\Phi_{i j}(p ; P, S)= & \sum_{\lambda} \frac{\delta\left((P-p)^{2}-m_{s}^{2}\right) \Theta\left(P^{0}-p^{0}\right)}{(2 \pi)^{3}}\left\langle P, S\left|\bar{\psi}_{j}(0) \mathcal{W}\left[0 \mid \infty, 0, \overrightarrow{0}_{T}\right]\right| d q ; P-p, \lambda\right\rangle \times \\
& \left\langle d q ; P-p, \lambda\left|\mathcal{W}\left[\infty, 0, \overrightarrow{0}_{T} \mid 0\right] \psi_{i}(0)\right| P, S\right\rangle .
\end{aligned}
$$

The essence of the diquark spectator model is to calculate the matrix elements in Eq. (1) by the introduction of effective The nucleon-diquark-quark vertices $\Upsilon_{s}(N)$ and $\Upsilon_{a x}^{\mu}(N)$ are represented in Fig. 1 (a). For example, the matrix element for the axial vector diquark is

$$
\left\langle a d q ; P-p ; \lambda\left|\psi_{i}(0)\right| P, S\right\rangle=i \frac{g_{a x}\left(p^{2}\right)}{\sqrt{3}} \varepsilon_{\mu}^{*}(P-p ; \lambda) \frac{\left[\left(\not p+m_{q}\right) \gamma_{5}\left[\gamma^{\mu}-R_{g} \frac{P^{\mu}}{M}\right] u(P, S)\right]_{i}}{p^{2}-m_{q}^{2}+i 0},
$$

where the polarization vector of the axial-vector diquark is $\varepsilon_{\mu}, u(P, S)$ denotes the nucleon spinor and $M$ and $m_{q}$ are nucleon and quark masses, respectively. The unpolarized TMD $f_{1}$ is obtained by inserting these expressions into Eq. (1) and projecting from the quark-quark correlator

$$
f_{1}\left(x, \vec{p}_{T}^{2}\right)=\left.\frac{1}{4} \int d p^{-}\left(\operatorname{Tr}\left[\gamma^{+} \Phi(p ; P, S)\right]+\operatorname{Tr}\left[\gamma^{+} \Phi(p ; P,-S)\right]\right)\right|_{p^{+}=x P^{+}},
$$

We have also calculated the distribution of transversely polarized quarks in a longitudinally polarized target, $h_{1 L}^{\perp}$ by replacing $\Gamma^{+}$with $\Gamma^{+} \Gamma^{i} \Gamma_{5}$ and $S$ by $S_{L}$, the spin 4 -vector in longitudinal direction. Their analytic expressions in the scalar and axial vector diquark sectors are given in [15].

In the spectator framework the "T-odd" TMDs [9] are generated by the gauge link $[10$, $6,7]$. The leading contribution, arising from the interference between tree- and box graph which contains an imaginary part necessary for "T-odds", is represented in Fig. 1 (c) in which the double line is an eikonal, and $l$ is the loop momentum. For an axial-vector diquark we model the composite nature of the diquark through an anomalous magnetic moment $\kappa$ [16]. In the notation of Fig. 1 (b) the gluon-diquark axial diquark vertex is

$$
\Gamma_{a x}^{\mu \nu_{1} \nu_{2}}=-i e_{d q}\left[g^{\nu_{1} \nu_{2}}\left(p_{1}+p_{2}\right)^{\mu}+(1+\kappa)\left(g^{\mu \nu_{2}}\left(p_{2}+q\right)^{\nu_{1}}+g^{\mu \nu_{1}}\left(p_{1}-q\right)^{\nu_{2}}\right)\right] .
$$

For $\kappa=-2$ the vertex $\Gamma_{a x}$ reduces to the standard $\gamma W W$-vertex. We express the matrix elements including the gauge link, $\left\langle d q ; P-p\left|\mathcal{W}\left[\infty, 0, \overrightarrow{0}_{T} \mid 0\right] \psi_{i}(0)\right| P, S\right\rangle$ in the one gluon 
approximation [15]. Projecting the Boer-Mulders function,

$$
\epsilon_{T}^{i j} p_{T}^{j} h_{1}^{\perp}\left(x, \vec{p}_{T}^{2}\right)=\frac{M}{4} \int d p^{-}\left(\operatorname{Tr}\left[\Phi_{\text {unpol }}(p, S) i \sigma^{i+} \gamma_{5}\right]+\operatorname{Tr}\left[\Phi_{\text {unpol }}(p,-S) i \sigma^{i+} \gamma_{5}\right]\right),
$$

where $\epsilon_{T}^{i j} \equiv \epsilon^{-+i j}$ the axial-vector diquark contribution is given by the expression,

$$
\begin{gathered}
\epsilon_{T}^{i j} p_{T}^{j} h_{1}^{\perp, a x}\left(x, \vec{p}_{T}^{2}\right)=-\frac{e_{q} e_{d q}}{8(2 \pi)^{3}} \frac{1}{\vec{p}_{T}^{2}+\tilde{m}^{2}} \frac{M}{P^{+}} \int \frac{d^{4} l}{(2 \pi)^{4}}\left\{\frac{1}{3} g_{a x}\left((l+p)^{2}\right) g_{a x}^{*}\left(p^{2}\right) \times\right. \\
\mathcal{D}_{\rho \eta}(P-p-l)\left(\sum_{\lambda} \varepsilon_{\sigma}^{*}(P-p ; \lambda) \varepsilon_{\mu}(P-p ; \lambda)\right) \times \\
\frac{\left[g^{\sigma \rho} v \cdot(2 P-2 p-l)+(1+\kappa)\left(v^{\sigma}(P-p+l)^{\rho}+v^{\rho}(P-p-2 l)^{\sigma}\right)\right]}{[l \cdot v+i 0]\left[l^{2}-\lambda^{2}+i 0\right]\left[(l+p)^{2}-m_{q}^{2}+i 0\right]} \times \\
\left.\operatorname{Tr}\left[(P+M)\left(\gamma^{\mu}-R_{g} \frac{P^{\mu}}{M}\right)\left(\not p-m_{q}\right) \gamma^{+} \gamma^{i}\left(y+\not p+m_{q}\right)\left(\gamma^{\eta}+R_{g} \frac{P^{\eta}}{M}\right) \gamma_{5}\right]\right\}+ \text { h.c. . }
\end{gathered}
$$

$\mathcal{D}(P-p-l)$ denotes the propagator of the axial-vector diquark. Since the numerator in Eq. (6) contains at most the loop momentum to the fourth power we can write it in the following manner, $\sum_{i=1}^{4} N_{\alpha_{1} \ldots \alpha_{i}}^{(i)} l^{\alpha_{1}} \ldots l^{\alpha_{i}}+N^{(0)}$. The (real) coefficients (tensors) $N_{\alpha_{1} \ldots \alpha_{i}}^{(i)}$ depend only on external momenta and can be computed in a straight-forward manner. The integration over the light cone components, $l^{+}$and $l^{-}$, are easily performed; however, calculating the $l^{+}$-integral results in an integral that is potentially ill-defined. This happens when $g\left(p^{2}\right)$ is a holomorphic function in $p^{2}$ and at least one of the Minkowski indices is light-like in the minus direction, e.g. $\alpha_{1}=-, \alpha_{2}, \ldots, \alpha_{i} \in\{+, \perp\}$ resulting in an integral of the form $\int d l^{+} \delta\left(l^{+}\right) \Theta\left(-l^{+}\right)$, implying that $l^{+}=0$ and $l^{-}=\infty$. This signals the existence of a light cone divergence in Ref. [12].

One can handle the light cone divergences by introducing phenomenological form factors with additional poles [15],

$$
g_{a x}\left(p^{2}\right)=\frac{\left(p^{2}-m_{q}^{2}\right) f\left(p^{2}\right)}{\left[p^{2}-\Lambda^{2}+i 0\right]^{n}} .
$$

For $n \geq 3$ there are enough powers of $l^{+}$to eliminate this divergence. $f\left(p^{2}\right)$ is a covariant Gaussian [15] which cuts off the $p_{T}$ integrations and $\Lambda$ is an arbitrary mass scale fixed by fitting $f_{1}$ to data. Similarly, the Sivers-function is projected from the trace of the quarkquark correlator (1) (see e.g. [17]),

$$
2 S_{T}^{i} \epsilon_{T}^{i j} p_{T}^{j} f_{1 T}^{\perp}\left(x, \vec{p}_{T}^{2}\right)=\left.\frac{M}{2} \int d p^{-}\left(\operatorname{Tr}\left[\gamma^{+} \Phi\left(p ; P, S_{T}\right)\right]-\operatorname{Tr}\left[\gamma^{+} \Phi\left(p ; P,-S_{T}\right)\right]\right)\right|_{p^{+}=x P^{+}} .
$$

It is well-known [6] that in the scalar diquark approximation the $h_{1}^{\perp}$ and $f_{1 T}^{\perp}$ coincide. By contrast the different Dirac structure for the chiral even $f_{1 T}^{\perp}$ and chiral odd $h_{1}^{\perp}$ in the axial-vector diquark sector, Eq. (5) and (8) respectively, lead to different coefficients in the decomposition $N_{\alpha_{1} \ldots \alpha_{i}}^{(i)}$ [15]. We fix most of the model parameters such as masses and normalizations by comparing the model result for the unpolarized $f_{1}$ for $u$ and $d$ quarks to the low-scale $\left(\mu^{2}=0.34 \mathrm{GeV}\right)$ data parameterization of GRV [18] see Fig. 2. Note that PDFs for $u$ and $d$ quarks are given by linear combinations of PDFs for an axial vector and 

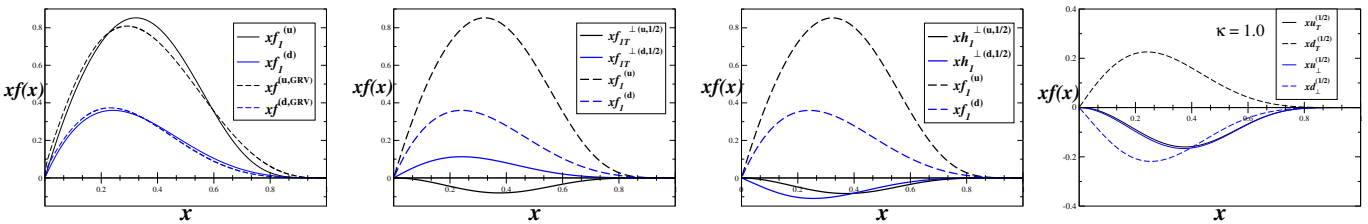

Figure 2: The unpolarized up and down quark distributions functions (left) versus $x$ compared to the low scale parameterization of GRV [18]. The half moment of the Sivers function (center-left) and Boer-Mulders function (center-right) and the unpolarized up and down quark distributions $(\kappa=1.0)$. The half-moment (left) and first moments (right) of the Boer-Mulders and Sivers functions versus $x$ fitted to extractions from data were presented in Ref. [19]

scalar diquark, $u=\frac{3}{2} f^{s c}+\frac{1}{2} f^{a x}$ and $d=f^{a x}[13,11]$. For "T-odd" PDFs we fix the sign and the strength of the final state interactions, the product of the charges of the diquark and quark, by comparing $f_{1 T}^{\perp}$ for $u$ and $d$ quarks in the diquark model with the existing data parameterizations (see Ref. [19]). The "one-half" moments $q_{T}^{(1 / 2)}$ (where $q=u, d$ ) of the Sivers and Boer-Mulders functions

$$
\frac{1}{2} q_{T}^{(1 / 2)}(x)=f_{1 T}^{\perp(q, 1 / 2)}(x)=\int d^{2} p_{T} \frac{\left|\vec{p}_{T}\right|}{2 M} f_{1 T}^{\perp(q)}\left(x, \vec{p}_{T}^{2}\right),
$$

are displayed along with the unpolarized $u$ and $d$ quark distributions in Fig. 2. The "onehalf" and first moments [15] of the up and down quark Sivers functions are negative and positive respectively while the up and down quark Boer-Mulders functions are both negative over the full range in Bjorken- $x$. We also note that the $u$-quark Sivers function and BoerMulders function are nearly equal, even with the inclusion of the axial vector spectator diquark.

Having explored the flavor dependence of the $h_{1}^{\perp}$ we are now in a position to extend early phenomenological work on "T-odd" contributions to azimuthal asymmetries in SIDIS[7]. We consider the spin independent double "T-odd" $\cos 2 \phi$ asymmetry for $\pi^{+}$and $\pi^{-}$production. We focus on the important contributions to the cross section for unpolarized SIDIS [17]

$$
\frac{d \sigma}{d x d y d z d \phi_{h} d P_{h \perp}^{2}} \approx \frac{2 \pi \alpha^{2}}{x y Q^{2}}\left[\left(1-y+\frac{1}{2} y^{2}\right) F_{U U, T}+(1-y) \cos \left(2 \phi_{h}\right) F_{U U}^{\cos 2 \phi_{h}}\right],
$$

where the structure function $F_{U U}^{\cos 2 \phi_{h}}$ involves a convolution of the Boer-Mulders and Collins fragmentation function

$$
F_{U U}^{\cos 2 \phi_{h}}=\mathcal{C}\left[-\frac{2 \hat{\boldsymbol{h}} \cdot \boldsymbol{k}_{T} \hat{\boldsymbol{h}} \cdot \boldsymbol{p}_{T}-\boldsymbol{k}_{T} \cdot \boldsymbol{p}_{T}}{M M_{h}} h_{1}^{\perp} H_{1}^{\perp}\right],
$$

$\mathcal{C}$ is the convolution integral. Our input for the Collins functions is based on recent work in [20] where the Collins function was calculated in the spectator framework. It was assumed that $H_{1}^{\perp(d i s-f a v)} \approx-H_{1}^{\perp(f a v)}$ in the pion sector, thereby satisfying the Schaefer-Teryaev sum rule [21] locally. We estimate the azimuthal asymmetry $A_{U U}^{\cos 2 \phi}$ (cf. Eq. (9)), where $A_{U U}^{\cos 2 \phi} \equiv \int \cos 2 \phi d \sigma / \int d \sigma$. In Fig. 3 we display the $A_{U U}^{\cos 2 \phi}\left(P_{T}\right)$ in the range of HERMES [3] and future JLAB kinematics [22] as well as $x$ dependence in the range $0.5<P_{T}<1.5 \mathrm{GeV} / \mathrm{c}$. 

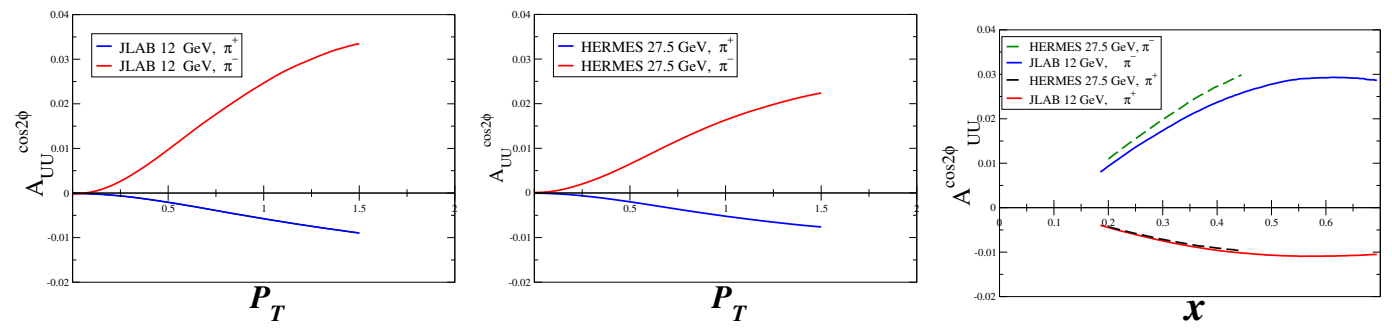

Figure 3: The $\cos 2 \phi$ asymmetry for and $\pi^{ \pm}$as a function of $P_{T}$ at JLAB $12 \mathrm{GeV}$ and HERMES (center) kinematics. Right: $\cos 2 \phi$ asymmetry for $\pi^{ \pm}$as a function of $x$ at JLAB $12 \mathrm{GeV}$ and HERMES kinematics.

Having calculated the chiral-odd but "T-even" parton distribution $h_{1 L}^{\perp}$ we use this result together with the result of Ref. [20] for the Collins function to give a prediction for the $\sin (2 \phi)$ single spin asymmetry $A_{U L}$ for a longitudinally polarized target. A decomposition into structure functions of the cross section of semi-inclusive DIS for a longitudinally polarized target reads (see e.g. [17])

$\frac{d \sigma_{U L}}{d x d y d z d \phi_{h} d P_{h \perp}^{2}} \approx \frac{2 \pi \alpha^{2}}{x y Q^{2}} S_{\|}\left[(1-y) \sin \left(2 \phi_{h}\right) F_{U L}^{\sin (2 \phi)}+(2-y) \sqrt{1-y} \sin \left(\phi_{h}\right) F_{U L}^{\sin \phi}\right]$,

$S_{\|}$is the projection of the spin vector on the direction of the virtual photon. In a partonic picture the structure function $F_{U L}^{\sin (2 \phi)}$ is a leading twist object (while $F_{U L}^{\sin \phi}$ is sub-leading) and given by a convolution of the TMD $h_{1 L}^{\perp}$ and the Collins function (cf. [17])

$$
F_{U L}^{\sin (2 \phi)}=\mathcal{C}\left[-\frac{2 \hat{\boldsymbol{h}} \cdot \boldsymbol{k}_{T} \hat{\boldsymbol{h}} \cdot \boldsymbol{p}_{T}-\boldsymbol{k}_{T} \cdot \boldsymbol{p}_{T}}{M M_{h}} h_{1 L}^{\perp} H_{1}^{\perp}\right] .
$$

We display the results for the single spin asymmetry $A_{U L}^{\sin (2 \phi)}$ in Fig. 4 using the kinematics of the upcoming JLab $12 \mathrm{GeV}$ upgrade. We note that the $\pi^{-}$asymmetry is large and positive due to the model assumption $H_{1}^{\perp(d i s-f a v)} \approx-H_{1}^{\perp(f a v)}$. This asymmetry has been measured at HERMES for longitudinally polarized protons [23] and deuterons [24]. The data show that for the proton target at HERMES $27.5 \mathrm{GeV}$ kinematics both $\pi^{+}$and $\pi^{-}$asymmetries are consistent with 0 down to a sensitivity of about 0.01 . These asymmetries could be non-zero, but with magnitudes less than 0.01 or 0.02 . These results are considerably smaller than our predictions for the JLab upgrade. For the deuteron target the results are consistent with 0 for $\pi^{+}$and $\pi^{-}$. This SIDIS data for polarized deuterons could reflect the near cancellation of $u$ - and $d$-quark $h_{1 L}^{\perp}$ functions and/or the large unfavored Collins function contributions. There is also CLAS preliminary data [25] at $5.7 \mathrm{GeV}$ that shows slightly negative asymmetries for $\pi^{+}$and $\pi^{-}$and leads to the extraction of a negative $x h_{1 L}^{\perp(u)}$. This suggests that the unfavored Collins function (for $d \rightarrow \pi^{+}$) is not contributing much. Data from the upgrade should help resolve these phenomenological questions.

We have performed calculations of transverse momentum dependent parton distributions, including the Boer-Mulders function $h_{1}^{\perp}$, the Sivers function $f_{1 T}^{\perp}$ along with $h_{1 L}^{\perp}$ in the 

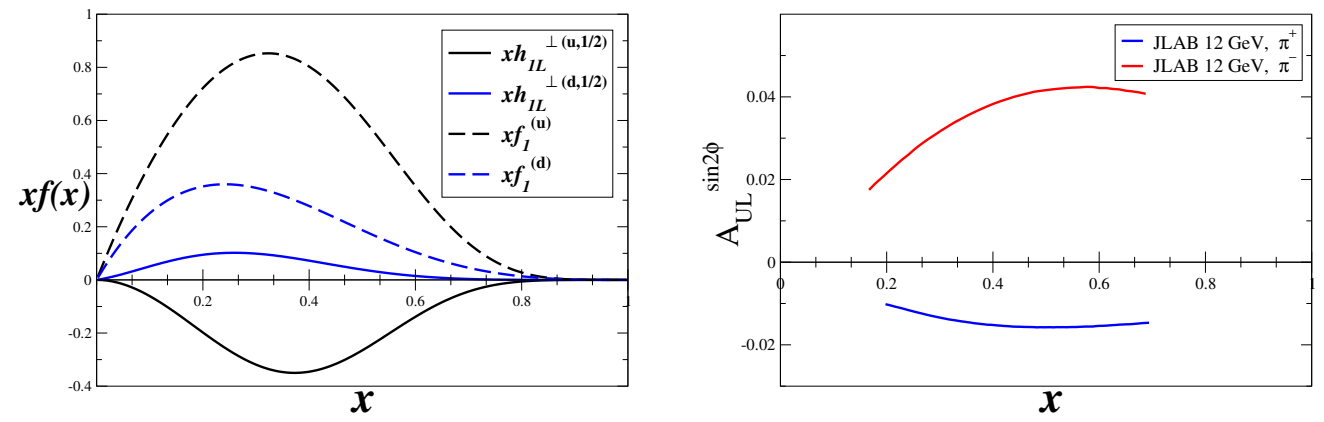

Figure 4: Left Panel: The half-moment of $x h_{1 L}^{\perp(1 / 2)}$ versus $x$ compared to the unpolarized up and down quark distribution functions. Right Panel: The $\sin 2 \phi$ asymmetry for $\pi^{+}$and $\pi^{-}$as a function of $x$ at JLAB $12 \mathrm{GeV}$ kinematics.

framework of an axial-vector and a scalar diquark spectator model. The calculation of these functions in both sectors allowed us to explore their flavor dependence, i.e. to compute $h_{1}^{\perp}$, $f_{1 T}^{\perp}$ and $h_{1 L}^{\perp}$ for a $u$-quark and a $d$-quark. We used these results along with the Collins fragmentation function $H_{1}^{\perp}$. to estimate the azimuthal asymmetries $A_{U U}^{(\cos (2 \phi))}$ and $A_{U L}^{(\sin (2 \phi))}$ in SIDIS. In summary, a refined diquark spectator model, including axial vector di-quarks leads to both $u$ - and $d$-quark "T-odd" TMDs and provides the ingredients for predicting a range of asymmetries for future experiments.

Acknowledgments

This work is supported in part by the U.S. Department of Energy under contracts, DEFG02-07ER41460 (LG), and DE-FG02-92ER40702 (GRG).

\section{References}

[1] Slides:

http: //indico. cern. ch/contributionDisplay. py? contribId=154\&sessionId=4\&c onf Id= 9499

[2] D. W. Sivers, Phys. Rev. D41, 83 (1990).

[3] HERMES, A. Airapetian et al., Phys. Rev. Lett. 94, 012002 (2005), hep-ex/0408013.

[4] COMPASS, V. Y. Alexakhin et al., Phys. Rev. Lett. 94, 202002 (2005), hep-ex/0503002.

[5] D. Boer and P. J. Mulders, Phys. Rev. D57, 5780 (1998), hep-ph/9711485.

[6] G. R. Goldstein and L. Gamberg, (2002), hep-ph/0209085.

[7] L. P. Gamberg, G. R. Goldstein, and K. A. Oganessyan, Phys. Rev. D67, 071504 (2003), hep$\mathrm{ph} / 0301018$.

[8] A. M. Kotzinian and P. J. Mulders, Phys. Lett. B406, 373 (1997), hep-ph/9701330.

[9] S. J. Brodsky, D. S. Hwang, and I. Schmidt, Phys. Lett. B530, 99 (2002), hep-ph/0201296.

[10] X.-d. Ji and F. Yuan, Phys. Lett. B543, 66 (2002), hep-ph/0206057.

[11] A. Bacchetta, A. Schaefer, and J.-J. Yang, Phys. Lett. B578, 109 (2004), hep-ph/0309246.

[12] L. P. Gamberg, D. S. Hwang, A. Metz, and M. Schlegel, Phys. Lett. B639, 508 (2006), hep-ph/0604022.

[13] R. Jakob, P. J. Mulders, and J. Rodrigues, Nucl. Phys. A626, 937 (1997), hep-ph/9704335.

[14] A. V. Belitsky, X. Ji, and F. Yuan, Nucl. Phys. B656, 165 (2003), hep-ph/0208038.

[15] L. P. Gamberg, G. R. Goldstein, and M. Schlegel, (2007), arXiv:0708.0324 [hep-ph].

[16] G. R. Goldstein and J. Maharana, Nuovo Cim. A59, 393 (1980).

[17] A. Bacchetta et al., JHEP 02, 093 (2007), hep-ph/0611265.

[18] M. Gluck, E. Reya, and A. Vogt, Eur. Phys. J. C5, 461 (1998), hep-ph/9806404. 
[19] M. Anselmino et al., (2005), hep-ph/0511017.

[20] A. Bacchetta, L. Gamberg, G. Goldstein, and A. Mukherjee, hep-ph/0707:3372.

[21] A. Schafer and O. V. Teryaev, Phys. Rev. D61, 077903 (2000), hep-ph/9908412.

[22] H. Avakian et al., approved JLab proposal PR 12-06-112. (2006).

[23] HERMES, A. Airapetian et al., Phys. Rev. Lett. 84, 4047 (2000), hep-ex/9910062.

[24] HERMES, A. Airapetian et al., Phys. Lett. B562, 182 (2003), hep-ex/0212039.

[25] CLAS, H. Avakian, P. E. Bosted, V. Burkert, and L. Elouadrhiri, AIP Conf. Proc. 792, 945 (2005), nucl-ex/0509032. 